\title{
Research on Semantic Interoperability for Business Collaboration
}

\author{
Zhan Jiang, Lanfen Lin, and Fei Xie \\ College of Computer Science \\ Zhejiang University \\ Hangzhou, China \\ \{jiangzhan, 1lf,skyfire_xiefei\}@zju.edu.cn
}

\begin{abstract}
In order to deal with the heterogeneous systems and variety of business data, business model and description of different enterprises in the interoperation process, a semantic interoperability framework for business collaboration is proposed in this paper. In the framework, a multi-facet ontology system supporting business collaboration is introduced to give unified understanding throughout interoperation process. Based on the framework, a method to extract ontologies from multi sources quickly and to realize semantic mapping based on mixed ontologies is utilized to resolve the semantic conflicts. Web service is employed to eliminate the system-level heterogeneity and is semantically enhanced to meet the requirements of service discovery. After that, the on-demand business construction is achieved through services dynamic combination. Finally, a prototype platform is developed and the feasibility of our framework was verified. Our proposed framework provides beneficial references for SMEs to realize inter-enterprise business collaboration in China.
\end{abstract}

Keywords: semantic interoperability (SI), ontology, Web service, business collaboration (BC).

\section{$1 \quad$ Introduction}

Software systems introduced by enterprises during the construction of enterprise informatization improve business efficiency. However, due to the heterogeneity caused by different software vendors and programming language, the business collaboration via system interoperation is hard to run smoothly. Web Service adopts a serial of standard technologies and protocols such as WSDL, UDDI, XML and SOAP [1]. Relevant business functions of enterprise systems are encapsulated into welldefined web services and invoked to shield system-level heterogeneity. Besides, it breaks through the restriction of territory and becomes a convenient and effective method to achieve long-distance business collaboration. Since enterprises differ in management pattern, business process and product model and so on, and employees in different departments or enterprises describe the business concept, data and model in dissimilar ways, semantic conflicts emerge throughout the process of business collaboration, it's necessary to find a method to resolve the semantic-level conflicts.

The original version of this chapter was revised: The copyright line was incorrect. This has been corrected. The Erratum to this chapter is available at DOI: 10.1007/978-3-642-33068-1_20

M. van Sinderen et al. (Eds.): IWEI 2012, LNBIP 122, pp. 201-208, 2012.

(C) IFIP International Federation for Information Processing 2012 
The emergence of ontology technology provides a new method for solving semantic problems of system interoperability. Ontology, as "an explicit specification of a conceptualization" [2], is able to represent the knowledge of specific domain in semantic level. It has been widely applied in many fields of computer science, such as knowledge sharing, software reusing and information retrieval. In order to achieve system interoperability among enterprises, ontology has been introduced by researchers to model heterogeneous systems to maintain semantic consistency among different systems. Bichindaritz proposes an ontology-based framework for semantic interoperability of case-based reasoning systems in biology and medicine [3]. Hu and Li etc. presents an ontology-based method of semantic interoperability to solve the interoperability problems in CAD and GIS [4]. Yang and Zhang gives a method of construction models interoperability by building construction domain ontology and modeling CAD models [5]. Park and Ram develop a comprehensive framework and methodology to facilitate semantic interoperability among distributed and heterogeneous information systems [6]. And lots of projects, likes STASIS, ONTOGOV, TERREGOV [7] also achieve semantic business collaboration in specific domain. Some researches concentrate in certain field and have domain limitations, and some are proposed in the general perspective and testified effectiveness. But it's hard to apply them to the SMEs collaborations in China. The informatization level of SMEs in China is backward, and there exists no available business model in these SMEs, not to mention ontology construction and maintenance. In this paper, taking SMEs in China as subject, considering the real situation encountered throughout business collaboration, a practical framework of semantic interoperability (SI) is proposed to realize business collaboration $(\mathrm{BC})$.

The rest of the paper is organized as follows. Section 2 describes the characteristics and obstacles of SMEs cooperation. The semantic interoperability framework is given in section 3. Section 4 elaborates on the key technologies involved during the process of business collaboration. Section 5 discusses the enterprise interoperation process and the implementation of the framework. Finally, section 6 concludes the paper and discusses some future work.

\section{Analysis of SMEs Collaboration}

There are five typical characteristics in the industry cluster of SMEs: 1)the large number of enterprises in the region, more than 90 percent of them are small and medium-sized enterprise(SME) . 2) The business collaboration in this area is extreme complex because of the large number of enterprises and the frequent business cooperation among them. The multiple collaborations among the enterprises include supply, manufacture, sales, logistics and service. 3) Among these SMEs, disparities of levels of IT are extraordinary. Not only the various software vendors, but also the incompatibility of information technologies like system architecture, infrastructure, programming languages and so on, leads to the high heterogeneity of systems, which means it is infeasible to realize system interaction by means of point-to-point system interface. 4) Enterprises benefit from the variety of product range but suffer from 
various product naming and coding rules. 5) The business process and marketing strategy are often changed to response to market fleetly.

Every enterprise cooperates with elses in one or more business fields by means of particular system. In the region, usually more than two enterprises participate in intercollaboration process. Taking account of product performance, price and delivery, the manufacturer could spill the order and purchase from a variety of sources. In this situation these systems of enterprises involved co-operate with others to realize the procurement. There are three obstacles existing in the business collaboration between enterprises:1) the business object model differs from system to system, and employee of different enterprises and departments differs in many aspects, like domain engaged, post occupied, education level, expression ability and so on, contributing to the different understanding and description of single concept. When enterprise cooperates with others, there might exist something ambiguous or even conflicting. 2) service adopts an open and standard method to shield the system-level heterogeneity, but the method only stipulate the specific grammar format of the interface, like service operations, inputs and outputs, lacking of semantic description of service features and functionality. Since the services aren't described accurately, it's difficult to meet the requirements of service discovery and combination. 3) the cooperative enterprise is chosen until the business collaboration begins, thus the inter-enterprise business process can't be preset. And enterprises differ in many aspects like business processes, organizations and management decisions, endowing business collaboration with the characteristics of high dynamic and randomness.

\section{The Framework of SI for BC}

In order to solve the problems of semantic inconsistency throughout the process of system interoperation, a multi-facet ontology system supporting business collaboration is introduced. The ontology system consists of shared top-level ontology, domain ontology, local ontology and service ontology. Shared top-level ontology, describing the general concept and the relationship between concepts, is domain-independent. It coordinates the relationship among else ontologies to maintain the concept consistency. Domain ontology and local ontology are both introduced to express the business concepts and relationships between concepts related in business collaboration. They differ in different objects, one for a specific domain and the other for concrete enterprise application. Service ontology is used to describe the concepts related with service and relationships. It gives the description of service attribute, functionality and interactive information. OWL-S [8] is adopted to describe the ontology information, since the model OWL-S of semantic description mainly focuses on the service functionality and is lack of service non-functional description. So the ServiceProfile ontoloty model of OWL-S is extended to express non-functional features like quality, expense, valuation. ServiceModel and ServiceGrouding are inherited to express basic attribute set and interface set.

Based on the building of ontology system, a framework of semantic interoperability for business collaboration is proposed, as shown in Fig. 1. There are 
three parts in the framework: the ontology system and the rules used for semantic conflicts resolution, the service repository based on the encapsulation of enterprise system and semantic enhancement, the dynamic combination of inter-enterprise process and the rules used for enterprise process conflicts resolution. The three parts dissolve the semantic inconsistency gradually in data level, system level and business process level corresponding to the obstacles met in the process of system interoperation. The three levels are progressive and the lower level of interoperability is the foundation of the upper level.

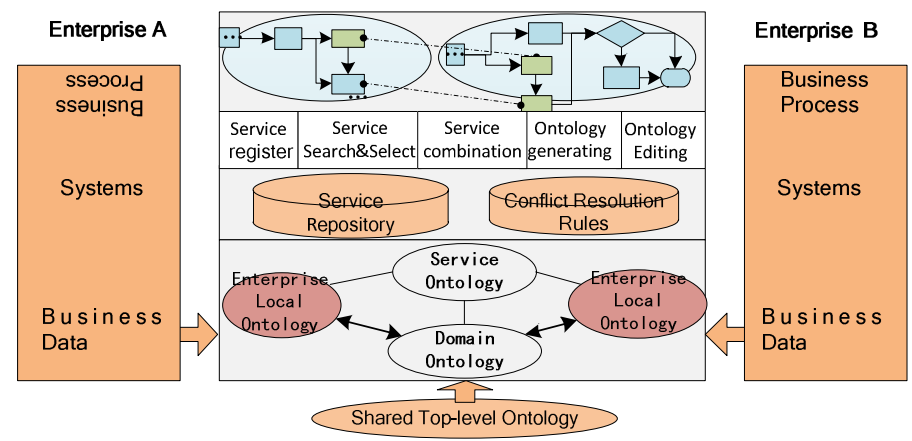

Fig. 1. The framework of semantic interoperability for business collaboration

\section{The Key Technologies of SI for BC}

\subsection{The Construction of Ontology for Business Collaboration}

The common method of building ontology [11] theoretically is correct, but the heavy workload and long cycle become prohibitive to SMEs in China, and it requires the ontology constructors having a good understanding of this domain. Consequently, a practical method for quickly building ontology is necessary.

\section{(1) Local Ontology Construction}

In the process of ontology construction on basis of structured data, the relational model (such as ER chart) can be obtained from database structure. The concepts, relationship, attributes, attribute type, primary key and foreign key can be extracted from the relational model and be transformed into corresponding rules, such as relationship are mapped to concept in the ontology, namely owl:Class, attribute are mapping to owl:Datatypeproperty, etc. These mapping rules are stored in the database for each enterprise. Under the guidance of the mapping rules, local ontology can be quickly built from databases of every enterprise.

Since there are a great number Semi-structured data, like XML files, which can be obtained from enterprise systems. Considering the regular corresponding relations between XML Schema and the description language of OWL [10,13], utilizing these XML files to construct local ontology appears feasible. Firstly, by parsing the XML 
document, extracting structural information and its data content, the corresponding XML Schema and XML Data files are generated respectively. Based on the detailed mapping relations between the elements of XML Schema and OWL [13], XSLT template is defined to transform the XML Schema elements to OWL elements. After the XML Data file is transformed to ontology instances, the ontology model and ontology instances are merged together to generate final ontology files.

Unstructured data is data without fixed structure and the method is similar with the common ontology construction method, it's too time-consuming and workload-heavy to build a local ontology in this way for an enterprise.

\section{(2) Domain Ontology Building}

Since the creation of domain ontology demands a mass of workload, and it's practically impossible for domain experts to finish it solely. The essence of Web 2.0 is using the internet to publish and share the information. Inspired by this, we utilize Web 2.0 technology to realize self-building of domain knowledge, only if a valid mechanism for information organization and management is adopted. In such case, the domain ontology is more effectual because it comes from the public and takes full advantage of the wisdom of them. This paper introduces a mature business domainindependent ontology as the basis, and the users in various regions can create and maintain the domain ontology by extending ontology content continuously, then domain experts or professionals review and normalize the concepts and knowledge enriched by different enterprise users, consequently forming a domain ontology shared by all users building.

\subsection{The Resolution of Semantic Conflicts in Enterprise Interoperability}

Semantic conflict can be divided into data-layer conflict and scheme-layer conflict. The former is caused by the different perception of the same concept and data value by different people, such as in naming, precision and representation, while the latter is caused by the differences of logic structure employed to express a same concept and data, including attribute set conflict, summarized conflict, polymerization conflict, associated conflict, etc. The process of semantic conflicts resolution is described below. First, the conflict list is generated by judging whether there are semantic conflicts exists between the collaborators. Then the mapping rules between the domain ontology and local ontologies are established for every collaborator respectively. After connecting the mapping rules of two collaborators together, the mapping specification is obtained. Thus, after the completion of the enterprise local ontology and domain ontology construction, how to establish the mapping between enterprise local ontology and domain ontology became a critical problem for the semantic conflicts resolution.

The multi-strategy mapping method first uses the enterprise local ontology and domain ontology as well as their respective data instances to train the learning classifier. According to classification results, a similarity measure algorithm is used to calculate the similarity of each pair of concept nodes, to determine the relative 
position of the concept in the other ontology. Then, based on HowNet, after using the method proposed by $\mathrm{Ni}$, Wang etc. [12] to calculate the similarity of concept definition and utilizing similarity evaluation function and to assess the similarity, the similarity matrix is gained. Finally knowledge of domain axiomatic constraints is applied to assess the similarity and the mapping rules are generated. The mapping relations, higher than threshold value settled are stored in the mapping rules library. Most of the data-layer and scheme-layer conflicts can be resolved through the mapping rules, also permitted to be created manually for others conflicts.

\subsection{On-Demand Inter-enterprise Business Processes Construction}

Since the inter-enterprise business process has the characteristics of high dynamic and randomness, service engine and service mediation are put forward to realize the service combination in two steps. First, requirements are annotated and divided into several structural sub-needs, describing detailed contents like product category, product name, quantity, delivery. The logic and constraint relations among the subneeds are recorded. Secondly, based on the description of sub-need, the service engine searches service repository to generate candidate services sets and evaluates the services combination. A multi-object genetic algorithm is employed to meet specific goals such as quality-preferred or delivery-preferred. After the optimal services portfolio are chosen, the relationship between requirements and service set is established. This process only concentrates on the temporal and logical relationships, hierarchical constraint of services from the functional and non-functional requirements of service, without considering the service provider, the actual business process. Next, service mediation analyze the actual process of every business interactions to generate the inter-enterprise business process. Finally, based on the relationship between requirements and service set and the inter-enterprise business process for each interaction, the on-demand inter-enterprise business processes is constructed by service mediations, as depicted in Fig. 2.

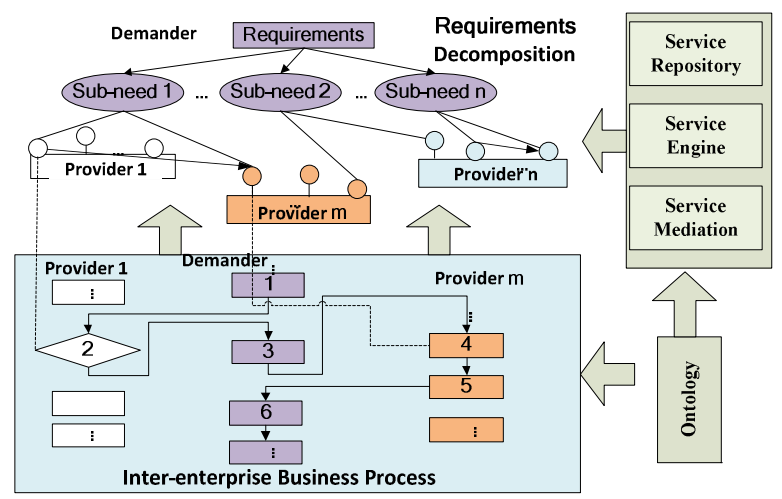

Fig. 2. On-demand inter-enterprise business processes construction 
Every enterprise needs to define its business processes, and the platform attempts to link the business processes of collaborative enterprise up to detect whether conflict exists. Once conflict is found, it implies related enterprises to resolve the conflicts by creating mapping rules or collaborative business process building. During the process of generating the inter-enterprise business process for each service interaction, owing to the different business process model and the description, the inter-enterprise business process should be constructed based on the business process mapping specification to resolve the inconsistency of business process. Based above, after dividing the requirements into sub-needs and mapping the sub-needs into service sets, the dynamic inter-enterprise business process is achieved by the combination of enterprise business process according to every service interaction of the service set.

\section{$5 \quad$ Application and Conclusion}

Based on the research contents, a platform based on J2EE framework, Web Services and OWL-S 1.1 specification is developed. The system mainly includes modules of ontology building and maintenance, service register center, business process model editing, conflict mapping rules management, workflow engine and so on. Table 1 shows the auto-generated mapping specification between the two enterprises' local ontologies. Fig. 3 shows an instantiation of the cross-enterprise business processes constructed. When manufacturer A publishes the demand as "products X5000 in 2-3 days" and chooses its suppliers, the platform detects the business process conflicts. Based on the mapping rules, both the supplier B and logistics services providers $\mathrm{C}$ add a new process as "send order confirmation" in order to comply with the process of A.

Table 1. The mapping specification between local ontologies

\begin{tabular}{lll}
\hline Local Ontology A & Domain general ontology & Local Ontology B \\
\hline Category: low- & Category: low-voltage & Category: low- \\
voltage apparatus & apparatus & voltage apparatus \\
Attribute :Pro-duct & Attribute :Product name & Attribute :Prod-uct \\
name & & description \\
Attribute: Product & Attribute: Product model & Attribute: Product \\
code & & NO. \\
Attribute: cost per & Attribute: unit cost & Attribute: unit \\
unit & & price \\
Attribute: number & Attribute: number & Attribute: quantity \\
\hline
\end{tabular}

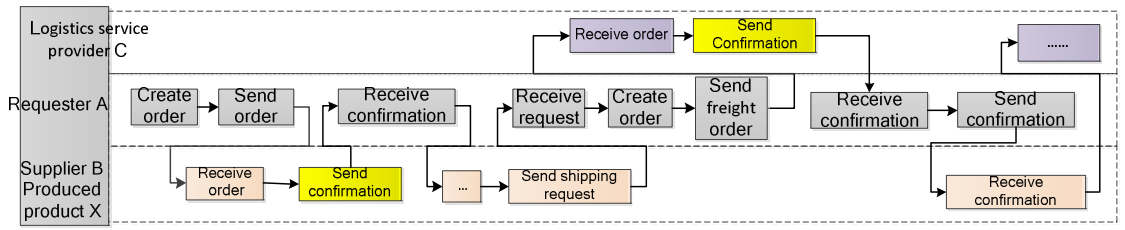

Fig. 3. Instantiation of inter-enterprise business process construction 
This platform has been partly applied to the representative Liushi low-voltage apparatus industry cluster in Zhejiang Province of China and proved to be workable. Through this platform, systems of different enterprises can interoperate with others and promote the efficiency of the business cooperation, especially among the core enterprise of the supply chain and the upstream and downstream enterprises surrounding it. But it still needs lots of related prerequisite work and manual intervention during the process of business collaboration, like business process model building, local ontology building, leading to the restriction of the application scope. The future work is to increase automation and reduce the workloads of enterprises.

\section{References}

1. Van Der Alsta, W.M.P., Benatallah, B., Casati, F., Curbera, F., et al.: Business process management: where business processes and web services meet. Data \&Knowledge Engineering 61(1), 1-5 (2007)

2. Uschold, M., King, M.: Towards a Methodology for Building Ontologies. In: Workshop on Basic Ontologies Issues in Knowledge Sharing (1995)

3. Bichindaritz, I.: Mémoire: A framework for semantic interoperability of case-based reasoning systems in biology and medicine. Artificial Intelligence in Medicine 36(2), 177-192 (2006)

4. Hu, Y.-J., Li, S.-P., Guo, M.: Ontology-based product knowledge representation. Journal of Computer-Aided Design \& Computer Graphics 15(12), 1531-1637 (2003) (in Chinese)

5. Yang, Q.Z., Zhang, Y.: Semantic interoperability in building design: methods and tools. Computer-Aided Design 38(10), 1099-1112 (2006)

6. Park, J., Ram, S.: Information systems interoperability: What lies beneath? ACM Transactions on Information Systems 22(4), 595-632 (2004)

7. Guglielmina, C.: The COIN Project - Vision, Objectives, Results. In: COIN IP Workshop. Enterprise Interoperability \& Collaboration in China: Barriers and Challenges, 20th Anniversary of the Cooperation between China and Europe in ICT for Enterprise

8. Jiang, Z., Wang, M., Lin, L.-F.: Research on service-oriented reconfigurable business collaboration platform for cluster supply chain. In: International Conference on Manufacturing Automation, ICMA (2010)

9. W3C. Team comment on the OWL-S submission[EB/OL] (September 12, 2007), http: / / www.w3 .org/Submission/2004/07/Comment

10. Rahm, E., Bernstein, P.A.: A survey of approaches to automatic schema matching. The VLDB Journal 10(4), 334-350 (2001)

11. Noy, N.F., McGuinness, D.L.: Ontology development 101: A guide to creating your first ontology. Technical Report SMI-2001-0880, Stanford Medical Informatics (2001)

12. Ni, Y., Wang, H., Huang, N., Lu, Y., et al.: A Heterogeneous System Integration Framework for Business Collaboration. In: Intelligent Computing and Intelligent Systems, ICIS (2009)

13. Bohring, H., Auer, S.: Mapping XML to OWL ontologies. In: Proceedings of 13 Leipziger Informatik-Tage (LIT 2005) Leipziger Germany. Lecture Notes in Informatics (LNI), Lecture Notes in Informatics (r2qi), pp. 21-23 (2005) 